\title{
Preliminary report: outbreak of Legionnaires' disease in the cities of Ulm and Neu-Ulm in Germany, December 2009 - January 2010
}

H von Baum (heike.von-baum@uniklinik-ulm.de) ${ }^{1}$, G Härter², A Essig ${ }^{1}$, C Lück ${ }^{3}$, T Gonser ${ }^{4}$, A Embacher ${ }^{4}$, S Brockmann 5

1. Institute of Medical Microbiology and Hygiene, Ulm University Hospitals, Germany

2. Department of Internal Medicine III, Infectious Diseases, Ulm University Hospitals, Germany

3. German Reference Laboratory for Legionella, Dresden University, Germany

4. Public Health Services Ulm and Neu-Ulm, Germany

5. Department of Epidemiology and Health Report, State Health Office, Stuttgart, Germany

Citation style for this article: von Baum H, Härter G, Essig A, Lück C, Gonser T, Embacher A, Brockmann S. Preliminary report: outbreak of Legionnaires' disease in the cities of Ulm and Neu-Ulm in Germany, December 2009- January 2010. Euro Surveill. 2010;15(4):pii=19472. Available online: http://www.eurosurveillance.org/ the cities

ViewArticle.aspx?Articleld $=19472$

This article has been published on 28 January 2010

Currently an investigation is ongoing to explore and control an outbreak of Legionnaires' disease, affecting 65 people as of 22 January 2010, in the cities of Ulm and Neu-Ulm, south-west Germany. A hitherto unidentified wet cooling system in these twin cities is considered as the most likely source of infection.

On 5 January 2010, Ulm University Hospital informed the local health office of a cluster of hospitalisations due to community acquired pneumonia caused by Legionella pneumophila serogroup (sg) 1. As of Friday 22 January 2010, 65 cases including five deaths were under investigation by the local and regional health authorities. With only a few exceptions all cases were living or working in Ulm or Neu-Ulm in south-west Germany. All cases are German residents aged between 27 and 96 years (median age 67 years) (Figure 1).

The majority of patients identified until 22 January 2010 had to be hospitalised, $n=61$. All patients and, if the clinical condition of the patient made an interview impossible, their family members, were interviewed using a standardised questionnaire to investigate potential sources of exposition, personal risk factors and the onset of symptoms. For 40 cases the onset of symptoms was during the last week of December 2009 (Figure 2). All but two patients who were admitted to our hospital had underlying diseases.

\section{Clinical findings}

In 41 of the patients who had been admitted to the University Hospital the following clinical symptoms were observed: fever in 35 of 41 patients, cough in 30 of 41 patients, abdominal pain and/or diarrhea in 11 of 41 patients, and symptoms relating to the central nervous system (confusion, somnolence or loss of consciousness, fainting) in 27 of 41 patients. All patients' chest radiographs showed an infiltrate. All patients were treated with standard antibiotic treatment consisting of a macrolid (clarithromycin), or preferably a fluoroquinolone (levofloxacin) for at least 14 days [1]. Four patients required mechanical ventilation. Four patients, two of whom had received mechanical ventilation and who had been treated in the University Hospital, died between several hours and eight days after admission. The other patients responded well to treatment and the majority recovered promptly. The median length of hospital stay of the patients, who were discharged until 22 January 2010, was 9.9 days (range 4-16 days).

\section{Laboratory investigations}

For the investigation of the outbreak, the case definitions of the European Working Group on Legionella Infection were applied. Considering clinical and laboratory criteria, all cases were classified as confirmed by positive results from laboratory tests for urinary antigen or culture [2]. Microbiological investigations of the majority of patients' specimens were performed in the Institute of Medical Microbiology and Hygiene, University Hospital of Ulm, Germany. Rapid testing of urine specimens for Legionella pneumophila sg 1 soluble antigen using an immunochromatographic card assay (Inverness Medical) was performed in patients with clinically suspected Legionnaires' disease. Results were confirmed by retesting concentrated urine specimens with an enzyme immunoassay (Biotest). Expectorated sputum samples and other respiratory secretions were plated onto buffered charcoal yeast agar. Colonies suspicious for Legionella were serotyped using a latex agglutination assay (Oxoid). In addition, respiratory samples were processed for detection of Legionella specific DNA by nucleic acid amplification. DNA was isolated from respiratory samples using the Magna pure system (Roche) and a L. pneumophila specific real-time LightCycler PCR was run targeting the macrophage infectivity potentiator (mip) gene as described previously [5]. 
Results

All patients with a microbiological workup in our laboratory had a positive urinary antigen test, in the respiratory material of 10 patients PCR for L. pneumophila was positive. Four clinical isolates belonging to sg1 were further subtyped by using monoclonal antibodies (MAb). All these isolates were identified as monoclonal subtype Knoxville (carrying the virulence associated epitope recognised by MAb 3-1 monoclonal antibody) [3]. Molecular identification of cultured legionellae was achieved by $16 \mathrm{~S}$ rRNA gene sequencing that showed a $99 \%$ homology to L. pneumophila in three isolates. So far, one isolate was genotyped and belongs to sequence type (ST) 62 [4].

\section{Epidemiological investigations and findings} Epidemiological and environmental investigations are coordinated by the local authorities in UIm and Neu-Ulm, with support of the state health offices in Baden-Württemberg and Bavaria. Investigations are

\section{FIGURE 1}

Age and sex distribution in patients with Legionnaires' disease, Ulm/Neu Ulm, Germany, as of 22 January 2010 $(n=65)$

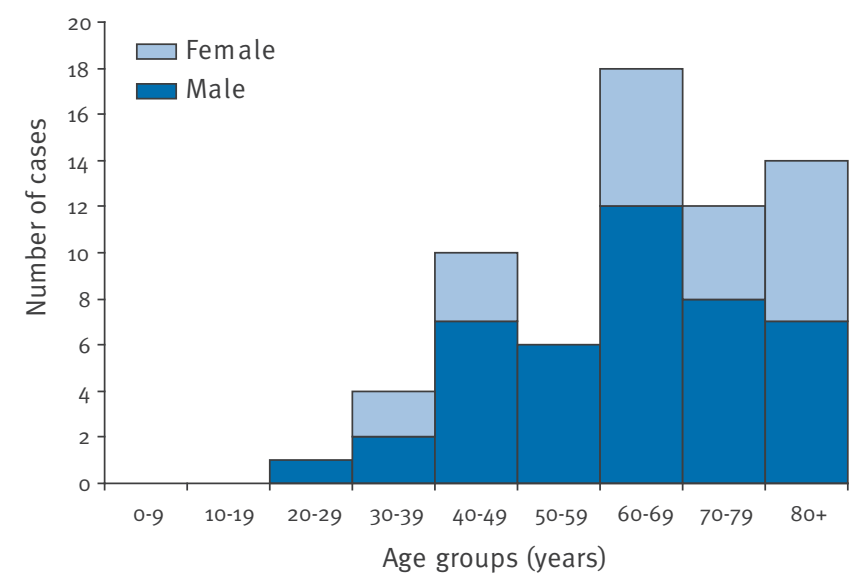

\section{FIGURE 2}

Onset of disease in patients with Legionnaires' disease, Ulm/Neu Ulm, Germany, information available as of 22 January 2010, $(n=46)$



in progress to identify the potential source of this outbreak by comparing environmental isolates from the patients' domestic installations and wet cooling systems from the areas of both cities with the clinical ones. According to the patient interviews, the cases had no common exposure to water supplies in public buildings, hotels, sport facilities or similar sources. Only living in, working in, or visiting Ulm or Neu-Ulm appeared as a common characteristic of the affected persons. Further epidemiological investigations are planned.

\section{Conclusions}

This is the largest community associated outbreak of Legionnaires' disease recognised in Germany so far. In dealing with the event several important steps in outbreak detection and management were confirmed: the importance of a rapid clinical diagnosis and thorough microbiological confirmation and the immediate involvement of the health authorities. The latter is necessary to initiate investigations to detect the source of the outbreak, to raise awareness of the problem in the community and to optimise communication of all involved parties. Furthermore we realised that an outbreak of Legionnaires' disease most likely related to wet cooling systems is not restricted to the warm seasons.

Hospitals, general practitioners and the public have been informed of the situation by the local authorities on a regular basis, starting on 5 January 2010. As of 23 January 2010, health authorities have no indication that persons from other countries have been affected. The last clinical case was hospitalised on 13 January 2010. We would be grateful for any reports of cases from other countries which could potentially be linked to this outbreak.

References

1. Höffken G, Lorenz J, Kern W, Welte T, Bauer T, Dalhoff $\mathrm{K}$, Dietrich E, Ewig S, Gastmeier P, Grabein B, Halle E, Kolditz M, Marre R, Sitter H; Paul-Ehrlich-Gesellschaft für Chemotherapie; Deutschen Gesellschaft für Pneumologie und Beatmungsmedizin; Deutschen Gesellschaft für Infektiologie und vom Kompetenznetzwerk CAPNETZ. Pneumologie. 2009 Oct;63(10):e1-68. Epub 2009 Oct 9. German.

2. European Working Group on Legionella Infections (EWGLI). Case definition. London: EWGLI. Available from: http://www. ewgli.org/data/european_guidelines/eg_appendix1.pdf

3. Helbig JH, Bernander S, Castellani Pastoris M, Etienne J, Gaia V, Lauwers S, et al. Pan-European study on culture-proven Legionnaires disease: distribution of Legionella pneumophila serogroups and monoclonal subgroups. Eur J Clin Microbiol Infect Dis 2002;21(10):710-6

4. Ratzow S, Gaia V, Helbig JH, Fry NK, Lück PC. Addition of neuA, the gene encoding $\mathrm{N}$-acylneuraminate cytidylyl transferase, increases the discriminatory ability of the consensus sequence-based scheme for typing Legionella pneumophila serogroup 1 strains. J Clin Microbiol 2007;45(6):1965-8

5. Wellinghausen N, Frost C, Marre R. Detection of legionellae in hospital water samples by quantitative real-time LightCycler PCR. Appl Environ Microbiol. 2001;67(9):3985-93 